\title{
On coefficients of vector-valued Bloch functions
}

\author{
by \\ Oscar Blasco (Valencia)
}

\begin{abstract}
Let $X$ be a complex Banach space and let $\operatorname{Bloch}(X)$ denote the space of $X$-valued analytic functions on the unit disc such that $\sup _{|z|<1}\left(1-|z|^{2}\right)\left\|f^{\prime}(z)\right\|<\infty$. A sequence $\left(T_{n}\right)_{n}$ of bounded operators between two Banach spaces $X$ and $Y$ is said to be an operator-valued multiplier between $\operatorname{Bloch}(X)$ and $\ell_{1}(Y)$ if the map $\sum_{n=0}^{\infty} x_{n} z^{n} \rightarrow$ $\left(T_{n}\left(x_{n}\right)\right)_{n}$ defines a bounded linear operator from $\operatorname{Bloch}(X)$ into $\ell_{1}(Y)$. It is shown that if $X$ is a Hilbert space then $\left(T_{n}\right)_{n}$ is a multiplier from $\operatorname{Bloch}(X)$ into $\ell_{1}(Y)$ if and only if $\sup _{k} \sum_{n=2^{k}}^{2^{k+1}}\left\|T_{n}\right\|^{2}<\infty$. Several results about Taylor coefficients of vector-valued Bloch functions depending on properties on $X$, such as Rademacher and Fourier type $p$, are presented.
\end{abstract}

1. Introduction. Throughout the paper $X$ stands for a complex Banach space and we write $\operatorname{Bloch}(X)$ for the space of $X$-valued analytic functions on the unit disc such that $\|f\|_{\operatorname{Bloch}(X)}=\|f(0)\|+\sup _{|z|<1}\left(1-|z|^{2}\right)\left\|f^{\prime}(z)\right\|<\infty$. We write Bloch instead of Bloch $(\mathbb{C})$.

Clearly, $f \in \operatorname{Bloch}(X)$ if and only if $x^{*} f(z)=\left\langle f(z), x^{*}\right\rangle \in$ Bloch for all $x^{*} \in X^{*}$ and $\|f\|_{\operatorname{Bloch}(X)} \approx \sup _{\left\|x^{*}\right\|=1}\left\|x^{*} f\right\|_{\text {Bloch }}$.

For $1 \leq p, q \leq \infty$ we denote by $\ell(p, q, X)$ the spaces of sequences $\left(x_{n}\right)_{n}$ in $X$ such that $\left(\left\|\left(\left\|x_{n}\right\|\right)_{n \in I_{k}}\right\|_{\ell_{p}}\right)_{k} \in \ell_{q}$, where $I_{k}=\left\{n \in \mathbb{N}: 2^{k-1} \leq n<2^{k}\right\}$ for $k \in \mathbb{N}$ and $I_{0}=\{0\}$. We write $\ell_{p}(X)$ for $\ell(p, p, X)$.

For $1 \leq p, q \leq \infty$ we write $\left\|\left(x_{n}\right)\right\|_{p, q}=\left\|\left(\left\|\left(\left\|x_{n}\right\|\right)_{n \in I_{k}}\right\|_{\ell_{p}}\right)_{k}\right\|_{\ell_{q}}$. As usual, when $X=\mathbb{C}$ we simply write $\ell(p, q)$. These classes were first introduced for the scalar-valued case by C. N. Kellogg in [20].

Let us recall the following well known fact on Taylor coefficients of Bloch functions. There exist $C_{1}, C_{2}>0$ such that

$$
C_{1}\left\|\left(x_{n}\right)\right\|_{\infty} \leq\|f\|_{\operatorname{Bloch}(X)} \leq C_{2}\left\|\left(x_{n}\right)\right\|_{1, \infty}
$$

for any $f(z)=\sum_{n=0}^{\infty} x_{n} z^{n}$ with $x_{n} \in X$.

2000 Mathematics Subject Classification: 46E40, 46B20.

Key words and phrases: Bloch functions, type and cotype.

Partially supported by Proyecto BMF2002-04013. 
Indeed, for each $n$ and $r \in(0,1)$,

$$
x_{n} r^{n}=\frac{1}{2 \pi} \int_{-\pi}^{\pi} f\left(r e^{i \theta}\right) e^{-i n \theta} d \theta .
$$

Hence $n\left\|x_{n}\right\| r^{n-1} \leq \sup _{|z|=r}\left\|f^{\prime}(z)\right\|$ for all $n \in \mathbb{N}$ and $0<r<1$. Now selecting $r=1-1 / n$ we obtain $\left\|\left(x_{n}\right)\right\|_{\infty} \leq C\|f\|_{\operatorname{Bloch}(X)}$. For the other inequality, observe that

$$
\left\|f^{\prime}(z)\right\| \leq \sum_{k} \sum_{n \in I_{k}} n\left\|x_{n}\right\||z|^{n-1} \leq\left\|\left(x_{n}\right)_{n}\right\|_{1, \infty} \sum_{k} 2^{k}|z|^{2^{k}-1} \leq C \frac{\left\|\left(x_{n}\right)_{n}\right\|_{1, \infty}}{1-|z|} .
$$

The reader is referred to $[1,2,6]$ for the general theory of Bloch functions.

Let $1 \leq p, q<\infty$. It is easy to see that $(\ell(p, q, X))^{*}=\ell\left(p^{\prime}, q^{\prime}, X^{*}\right)$ for $1 / p+1 / p^{\prime}=1 / q+1 / q^{\prime}=1$, under the natural pairing

$$
\left\langle\left(x_{n}\right),\left(x_{n}^{*}\right)\right\rangle=\sum_{n}\left\langle x_{n}, x_{n}^{*}\right\rangle
$$

(where we also use $\langle\cdot, \cdot\rangle$ for the dual pairing in $X$ ). Due to the fact that we would like to identify the analytic functions with the sequences corresponding to their Taylor coefficients, it is convenient to find a predual of $\operatorname{Bloch}\left(X^{*}\right)$ under the previous pairing.

We shall be denoting by $J_{1}(X)$ the space of $X$-valued analytic functions $f$ on the disc $\mathbb{D}$ such that $\int_{0}^{1} M_{1}\left(f^{\prime}, r\right) d r<\infty$, where

$$
M_{p}(f, r)=\left(\int_{0}^{2 \pi}\left\|f\left(e^{i t}\right)\right\|^{p} \frac{d t}{2 \pi}\right)^{1 / p} \quad \text { for } 1 \leq p \leq \infty .
$$

Endowing the space with the norm $\|f\|_{J_{1}(X)}=\|f(0)\|+\int_{0}^{1} M_{1}\left(f^{\prime}, r\right) d r$ one gets $\left(J_{1}(X)\right)^{*}=\operatorname{Bloch}\left(X^{*}\right)$ under the pairing

$$
\langle f, g\rangle=\sum_{n=0}^{\infty}\left\langle x_{n}^{*}, x_{n}\right\rangle
$$

for any $g(z)=\sum_{n=0}^{\infty} x_{n}^{*} z^{n} \in \operatorname{Bloch}\left(X^{*}\right)$ and $f(z)=\sum_{n=0}^{\infty} x_{n} z^{n} \in J_{1}(X)$.

The reader is referred to [1] for this duality result in the scalar-valued case and to $[7,8]$ for its vector-valued extension. Another predual can be obtained in terms of Bergman spaces, namely $\left(A_{1}(X)\right)^{*}=\operatorname{Bloch}\left(X^{*}\right)$ (see $[27,5]$ ), where $A_{1}(X)$ denotes the space of $X$-valued analytic functions $f$ on the disc $\mathbb{D}$ such that $\int_{\mathbb{D}}\|f(z)\| d A(z)<\infty$ and $d A(z)$ stands for the normalized area measure on $\mathbb{D}$, although in this duality the pairing is different from (2).

Hence from (1) and (3) we can conclude that there exist $C_{1}, C_{2}>0$ such that

$$
C_{1}\left\|\left(x_{n}\right)\right\|_{\infty, 1} \leq\|f\|_{J_{1}(X)} \leq C_{2}\left\|\left(x_{n}\right)\right\|_{1}
$$

for any $f \in J_{1}(X)$ with Taylor coefficients $\left(x_{n}\right)$. 
Vector-valued Bloch functions have been used in different papers and for different reasons (see $[3,4,7-12]$ ). We refer the reader to $[5,13]$ for new results on the subject.

In this paper we shall deal with the vector-valued analogues of the following result on multipliers due to J. M. Anderson and A. L. Shields (see [2]):

$$
\text { (Bloch, } \left.\ell_{1}\right)=\ell(2,1),
$$

where (Bloch, $\left.\ell_{1}\right)$ stands for the space of sequences $\lambda=\left(\lambda_{n}\right)$ such that the operator $T_{\lambda}(f)=\left(\lambda_{n} \alpha_{n}\right)_{n}$ for $f(z)=\sum_{n} \alpha_{n} z^{n}$ is bounded from Bloch into $\ell_{1}$.

As a consequence of (5) one gets the following improvement of (1): There exists a constant $C>0$ such that

$$
\left\|\left(\alpha_{n}\right)_{n}\right\|_{2, \infty} \leq C\|\phi\|_{\text {Bloch }}
$$

for any $\phi(z)=\sum_{n=0}^{\infty} \alpha_{n} z^{n}$. We first observe that (6) does not hold in the vector-valued situation. Note that if $e_{n}$ stands for the canonical basis of $c_{0}$ then $f(z)=\sum_{n=1}^{\infty} e_{n} z^{n}=\left(z^{n}\right)_{n}$ is a bounded $c_{0}$-valued analytic function. In particular $f \in \operatorname{Bloch}\left(c_{0}\right)$, and $\left(e_{n}\right) \notin \ell\left(p, \infty, c_{0}\right)$ for any $p<\infty$. Hence (5) does not hold for general Banach spaces.

The aim of this paper is to understand whether (6) and (5) have natural extensions to vector-valued functions and how their vector-valued analogues depend on some geometrical properties of the Banach space $X$.

Problem 1. For which Banach spaces $X$ does the following hold:

$$
f(z)=\sum_{n=0}^{\infty} x_{n} z^{n} \in \operatorname{Bloch}(X) \Rightarrow\left(x_{n}\right)_{n} \in \ell(2, \infty, X) ?
$$

Let us give the following definition.

Definition 1.1. Let $X$ be a complex Banach space. Define $\Lambda_{\mathrm{Bloch}, \ell_{1}}(X)$ as the space of scalar-valued sequences $\lambda=\left(\lambda_{n}\right)_{n}$ such that the operator $T_{\lambda}(f)=\left(\lambda_{n} x_{n}\right)_{n}$ for $f(z)=\sum_{n=0}^{\infty} x_{n} z^{n}$ is bounded from $\operatorname{Bloch}(X)$ into $\ell_{1}(X)$.

Obviously, taking $f(z)=x \phi(z)$ where $x \in X$ and $\phi \in$ Bloch one gets $\Lambda_{\text {Bloch }, \ell_{1}}(X) \subseteq\left(\right.$ Bloch,$\left.\ell_{1}\right)=\ell(2,1)$.

A dual argument shows that, for $1<p \leq 2$, the inequality

$$
\left\|\left(x_{n}\right)_{n}\right\|_{p^{\prime}, \infty} \leq C\left\|\sum x_{n} z^{n}\right\|_{\operatorname{Bloch}(X)}
$$

is equivalent to

$$
\ell(p, 1) \subseteq \Lambda_{\text {Bloch }, \ell_{1}}(X) .
$$

Hence Problem 1 can be rephrased as follows: For which Banach spaces $X$, $\Lambda_{\text {Bloch }, \ell_{1}}(X)=\ell(2,1)$ ? 
The example given after (6) shows that $\ell(p, 1)$ in not contained in $\Lambda_{\text {Bloch, } \ell_{1}}\left(c_{0}\right)$ for any $p>1$. This actually leads to a more general question.

Problem 2. Find $\Lambda_{\mathrm{Bloch}, \ell_{1}}(X)$ for a given Banach space $X$.

Similar problems and descriptions for vector-valued Hardy and Bergman spaces were considered in previous papers by the author (see [4, 14, 15]).

Another possible generalization of (5) is to consider sequences of bounded operators $\left(T_{n}\right)_{n}$ in $\mathcal{L}(X, Y)$ between two Banach spaces $X$ and $Y$ and to define operator-valued multipliers. This approach for different spaces of analytic functions and multipliers can be found in $[3,4,9,10,12,13]$.

Definition 1.2. A sequence $\left(T_{n}\right)_{n}$ in $\mathcal{L}(X, Y)$ is said to be a multiplier between $\operatorname{Bloch}(X)$ and $\ell_{1}(Y)$, written $\left(T_{n}\right) \in\left(\operatorname{Bloch}(X), \ell_{1}(Y)\right)$, if $\left(T_{n}\left(x_{n}\right)\right)_{n}$ belongs to $\ell_{1}(Y)$ whenever $f(z)=\sum_{n=0}^{\infty} x_{n} z^{n}$ belongs to $\operatorname{Bloch}(X)$. This is equivalent to the existence of a constant $C>0$ such that

$$
\sum_{n=0}^{N}\left\|T_{n}\left(x_{n}\right)\right\| \leq C \sup _{|z|<1}\left(1-|z|^{2}\right)\left\|\sum_{n=1}^{N} n x_{n} z^{n-1}\right\|
$$

for any $N \in \mathbb{N}$ and $x_{0}, x_{1}, \ldots, x_{N} \in X$.

The infimum of the constants $C$ satisfying (8) is the multiplier norm, which coincides with the norm of $\Phi_{T}\left(\sum x_{n} z^{n}\right)=\left(T_{n}\left(x_{n}\right)\right)$ as the operator from $\operatorname{Bloch}(X)$ and $\ell_{1}(Y)$.

We shall address in the paper some partial answers to the more general problem of finding conditions on the Banach spaces $X$ and $Y$ to have

$$
\left(\operatorname{Bloch}(X), \ell_{1}(Y)\right)=\ell(2,1, \mathcal{L}(X, Y)) .
$$

Let us now collect several definitions of properties of Banach spaces to be used in what follows.

Definition 1.3. Let $1 \leq p \leq 2 \leq q<\infty$ and let $X$ be a complex Banach space. $X$ is said to have Fourier type $p$ if there exists a constant $C$ such that

$$
\left(\sum_{n=-\infty}^{\infty}\|\hat{f}(n)\|^{p^{\prime}}\right)^{1 / p^{\prime}} \leq C\|f\|_{L^{p}(\mathbb{T}, X)}
$$

for all functions $f \in L^{p}(\mathbb{T}, X)$.

$X$ is said to have Rademacher type $p$, resp. Rademacher cotype $q$, if there exists a constant $C$ such that

$$
\int_{0}^{1}\left\|\sum_{j=1}^{n} x_{j} r_{j}(t)\right\| d t \leq C\left(\sum_{j=1}^{n}\left\|x_{j}\right\|^{p}\right)^{1 / p},
$$


resp.

$$
\left(\sum_{j=1}^{n}\left\|x_{j}\right\|^{q}\right)^{1 / q} \leq C \int_{0}^{1}\left\|\sum_{j=1}^{n} x_{j} r_{j}(t)\right\| d t
$$

for any finite family $x_{1}, \ldots, x_{n}$ of vectors in $X$ where $r_{j}$ stand for the Rademacher functions on $[0,1]$.

The notion of Fourier type was first introduced by J. Peetre ([23]) and we refer the reader to the survey [18] for a complete study and references about this property. We just mention here that $X$ has Fourier type $p$ if and only if $X^{*}$ does. In particular, if $X$ has Fourier type $p$ then

$$
\|f\|_{L^{p^{\prime}}(\mathbb{T}, X)} \leq C\left(\sum_{n=-\infty}^{\infty}\|\widehat{f}(n)\|^{p}\right)^{1 / p} .
$$

The notions of Rademacher type and cotype were introduced by B. Maurey and G. Pisier (see $[16,22,26]$ ) and were shown to be rather important in Banach space theory. Let us simply recall that Fourier type $p$ implies Rademacher type $p$ and that if $X^{*}$ has type $p$ then $X$ has cotype $p^{\prime}$.

The main examples of spaces of Fourier type $p$ are $L^{r}(\mu)$ for any $p \leq r$ $\leq p^{\prime}$ or interpolation spaces $\left[X_{0}, X_{1}\right]_{\theta}$ between any Banach space $X_{0}$ and any Hilbert space $X_{1}$ where $1 / p=1-\theta / 2$.

Recall also that $L^{r}(\mu)$ has Rademacher type $\min \{p, 2\}$ and Rademacher cotype $\max \{p, 2\}$.

2. Taylor coefficients. We start by mentioning a couple of examples of vector-valued Bloch functions to be used later on.

ExAMPLE 2.1 (see [13, Example 3.1]). Let $1 \leq p \leq \infty$ and define $f_{p}$ : $\mathbb{D} \rightarrow \ell_{p}$ by $f_{p}(z)=\sum_{n=1}^{\infty} n^{-1 / p} e_{n} z^{n}$, where $e_{n}$ stands for the canonical basis. Then $f_{p} \in \operatorname{Bloch}\left(\ell_{p}\right)$.

Note that $f_{p}(z)=\sum_{n=1}^{\infty} x_{n} z^{n}$ with $\left\|x_{n}\right\|=n^{-1 / p}$ and that $\left(x_{n}\right) \in$ $\ell\left(2, \infty, \ell_{p}\right)$ if and only if $p \geq 2$.

ExAMPLE 2.2 (see [13, Example 3.2]). Let $1 \leq p<\infty$ and define $F_{p}$ : $\mathbb{D} \rightarrow L^{p}(\mathbb{T})$ by $F_{p}(z)(\xi)=(1-\bar{\xi} z)^{-1 / p}$. Then $F_{p} \in \operatorname{Bloch}\left(L^{p}(\mathbb{T})\right)$.

Note that $F_{p}(z)=\sum_{n=1}^{\infty} x_{n}^{\prime} z^{n}$ with $\left\|x_{n}^{\prime}\right\| \approx n^{-1 / p^{\prime}}$ and that $\left(x_{n}\right) \in$ $\ell\left(2, \infty, L^{p}(\mathbb{T})\right)$ if and only if $p \leq 2$.

These examples show that

$$
\Lambda_{\text {Bloch }, \ell_{1}}\left(\ell_{p}\right) \subsetneq \ell(2,1) \quad \text { for } p<2, \quad \Lambda_{\text {Bloch }, \ell_{1}}\left(L^{p}(\mathbb{T})\right) \subsetneq \ell(2,1) \text { for } p>2 \text {. }
$$

We now show that (7) holds for Hilbert spaces. The proof that we shall present is based upon Grothendieck's inequality. 
THEOREM 2.1. Let $H$ be a Hilbert space. Then there exists a constant $C>0$ such that

$$
\left\|\left(x_{n}\right)_{n}\right\|_{2, \infty} \leq C\|f\|_{\operatorname{Bloch}(H)}
$$

for all $f(z)=\sum_{n=0}^{\infty} x_{n} z^{n} \in \operatorname{Bloch}(H)$. Hence $\Lambda_{\mathrm{Bloch}, \ell_{1}}(H)=\ell(2,1)$.

Proof. Given $f \in \operatorname{Bloch}(H)$ we start by defining $T_{f}: A_{1} \rightarrow H$ by the formula $T_{f}\left(u_{n}\right)=x_{n}$, where $u_{n}(z)=(n+1) z^{n}$, and extending the definition to all polynomials by linearity. That is,

$$
T_{f}(\phi)=\sum_{n} \frac{x_{n} \alpha_{n}}{n+1}=\int_{\mathbb{D}} \phi(\bar{z}) f(z) d A(z)
$$

for $\phi(z)=\sum_{n=0}^{N} \alpha_{n} z^{n}$.

Using the fact that

$$
\langle\phi, \psi\rangle=\sum_{n=0}^{\infty} \frac{\alpha_{n} \bar{\beta}_{n}}{n+1}=\int_{\mathbb{D}} \phi(z) \overline{\psi(z)} d A(z),
$$

for any $\phi(z)=\sum_{n=0}^{N} \alpha_{n} z^{n}$ and $\psi(z)=\sum_{n=0}^{\infty} \beta_{n} z^{n}$, gives the duality $\left(A_{1}\right)^{*}=$ Bloch (see [27]), together with the facts that $\left\langle T_{f}(\phi), x^{*}\right\rangle=\left\langle x^{*} f, \phi\right\rangle$ and polynomials are dense in $A_{1}$, we can continuously extend $T_{f}$ to $A_{1}$ as a bounded operator and $\left\|T_{f}\right\| \leq C\|f\|_{\operatorname{Bloch}(H)}$.

On the other hand it is known (see [26] or [27]) that $A_{1}$ is isomorphic to $\ell_{1}$. Hence by invoking the Grothendieck theorem (see [16]) we deduce that $T_{f}$ is absolutely summing.

Let $\left\|\left(\lambda_{n}\right)\right\|_{2,1} \leq 1$. It follows from (5) that

$$
\sup _{\|g\|_{\left(A_{1}\right)^{*} \leq 1}} \sum_{n}\left|\left\langle\lambda_{n} u_{n}, g\right\rangle\right| \leq C .
$$

This leads to

$$
\sum_{n}\left|\lambda_{n}\right|\left\|T\left(u_{n}\right)\right\| \leq C
$$

for all $\left\|\left(\lambda_{n}\right)\right\|_{2,1} \leq 1$. Or in other words $\left(x_{n}\right) \in \ell(2, \infty, X)$ and

$$
\left\|\left(x_{n}\right)_{n}\right\|_{2, \infty} \leq C\left\|T_{f}\right\|=C\|f\|_{\operatorname{Bloch}(H)} \text {. }
$$

We shall try to see how some geometrical properties of the space $X$ help to describe $\Lambda_{\mathrm{Bloch}, \ell_{1}}(X)$.

We first improve the estimates in (4) under some assumptions on the Banach space $X$. To do that we use the following lemma.

LEMma 2.2 (see [11] or [21]). Let $\left(\alpha_{n}\right)$ be a sequence of nonnegative numbers and $0<q, \beta<\infty$. Then

$$
\int_{0}^{1}(1-r)^{\beta q-1}\left(\sum_{n=1}^{\infty} \alpha_{n} r^{n}\right)^{q} d r \approx \sum_{k=1}^{\infty}\left(\sum_{n \in I_{k}} \frac{\alpha_{n}}{n^{\beta}}\right)^{q} .
$$


TheOREM 2.3. Let $1 \leq p \leq 2$ and $X$ be a Banach space of Fourier type $p$.

(i) There exists a constant $C>0$ such that

$$
\begin{gathered}
\|f\|_{J_{1}(X)} \leq C\left\|\left(x_{n}\right)\right\|_{p, 1} \\
\text { for all }\left(x_{n}\right) \in \ell(p, 1, X) \text { and } f(z)=\sum_{n=1}^{\infty} x_{n} z^{n} .
\end{gathered}
$$

(ii) There exists a constant $C>0$ such that

$$
\left\|\left(x_{n}\right)\right\|_{p^{\prime}, \infty} \leq C\|f\|_{\operatorname{Bloch}(X)}
$$

for all $f(z)=\sum_{n=1}^{\infty} x_{n} z^{n} \in \operatorname{Bloch}(X)$.

Proof. (i) Note that, by (11),

$$
\|f\|_{J_{1}(X)} \leq\|f(0)\|+\int_{0}^{1} M_{p^{\prime}}\left(f^{\prime}, r\right) d r \leq C\left(\|f(0)\|+\int_{0}^{1}\left(\sum_{n} n^{p}\left\|x_{n}\right\|^{p} r^{n p}\right)^{1 / p} d r\right) .
$$

Now apply Lemma 2.2 for $\beta=p$ and $q=1 / p$ to get $\|f\|_{J_{1}(X)} \leq C\left\|\left(x_{n}\right)\right\|_{p, 1}$.

(ii) Using the fact that $\operatorname{Bloch}(X)$ is isometrically included in $\left(J_{1}\left(X^{*}\right)\right)^{*}$ together with (i) and the fact that $X^{*}$ also has Fourier type $p$ one gets, for $f(z)=\sum_{n=1}^{\infty} x_{n} z^{n}$

$$
\begin{aligned}
\left\|\left(x_{n}\right)\right\|_{p^{\prime}, \infty} & =\sup \left\{\sum_{n}\left\langle x_{n}, x_{n}^{*}\right\rangle:\left\|\left(x_{n}^{*}\right)\right\|_{p, 1}=1\right\} \\
& \leq C \sup \left\{\langle f, g\rangle:\|g\|_{J_{1}\left(X^{*}\right)}=1\right\} \leq C\|f\|_{\operatorname{Bloch}(X)} .
\end{aligned}
$$

Theorem 2.4. Let $1<p<2$ and let $X$ be a Banach space.

(i) If $\ell(p, 1) \subseteq \Lambda_{\mathrm{Bloch}, \ell_{1}}(X)$ then $X$ has cotype $p^{\prime}$.

(ii) If $\ell(2,1)=\Lambda_{\mathrm{Bloch}, \ell_{1}}(X)$ then $X$ has Orlicz property, i.e. there exists $C>0$ so that $\left(\sum_{n}\left\|x_{n}\right\|^{2}\right)^{1 / 2} \leq C \sup _{\left\|x^{*}\right\|=1} \sum_{n}\left|\left\langle x_{n}, x^{*}\right\rangle\right|$.

Proof. We shall see in both cases that $\ell(p, 1) \subseteq \Lambda_{\mathrm{Bloch}, \ell_{1}}(X)$ implies that if $\sup _{\left\|x^{*}\right\|=1} \sum_{n}\left|\left\langle x_{n}, x^{*}\right\rangle\right|<\infty$ then $\sum_{n}\left\|x_{n}\right\|^{p^{\prime}}<\infty$. This, in the case $p<2$, is equivalent to $X$ having cotype $p^{\prime}$ (see $[24,25]$ ).

Let $x_{1}, \ldots, x_{N} \in X$ be such that $\sup _{\left\|x^{*}\right\|=1} \sum_{n=1}^{N}\left|\left\langle x_{n}, x^{*}\right\rangle\right|=1$. Take $k$ such that $2^{k-1} \leq N<2^{k}$ and construct $f(z)=\sum_{n=2^{k}+1}^{2^{k}+N} x_{n-2^{k}} z^{n}$. Hence $f$ belongs to $\operatorname{Bloch}(X)$ (because $x^{*} f \in$ Bloch for all $x^{*} \in X^{*}$ ). Therefore $\sum_{k=1}^{N}\left\|\lambda_{n} x_{n}\right\| \leq C$ for all $\left(\lambda_{n}\right)$ such that $\left\|\left(\lambda_{n}\right)_{n \in I_{k}}\right\|_{p}=1$. Hence $\sum_{n=1}^{N}\left\|x_{n}\right\|^{p^{\prime}} \leq C$.

Corollary 2.5. Let $X$ be a Banach space and $1 \leq p \leq 2$. Then $X$ has Fourier type $p \Rightarrow \ell(p, 1) \subseteq \Lambda_{\text {Bloch }, \ell_{1}}(X) \Rightarrow X$ has cotype $p^{\prime}$. 
3. Multipliers. Now we analyze the interplay between geometry of Banach spaces and questions (7) and (9).

Repeating the argument in Theorem 2.4 with $T_{n}=\lambda_{n} T$ for a fixed operator $T$ we obtain the following result.

Proposition 3.1. Let $1 \leq p \leq 2$ and let $X$ and $Y$ be Banach spaces. If

$$
\ell(p, 1, \mathcal{L}(X, Y)) \subseteq\left(\operatorname{Bloch}(X), \ell_{1}(Y)\right)
$$

then $\Pi_{p^{\prime}, 1}(X, Y)=\mathcal{L}(X, Y)$, where $\Pi_{p^{\prime}, 1}(X, Y)$ stands for the space of $\left(p^{\prime}, 1\right)$ summing operators (see [16]).

Proposition 3.2. Let $X$ and $Y$ be Banach spaces and assume that $X$ has Fourier type $p$. Then

$$
\ell(p, 1, \mathcal{L}(X, Y)) \subseteq\left(\operatorname{Bloch}(X), \ell_{1}(Y)\right) .
$$

Proof. This follows easily from Theorem 2.3 , since

$$
\sum_{n=1}^{\infty}\left\|T_{n}\left(x_{n}\right)\right\| \leq\left\|\left(T_{n}\right)\right\|_{p, 1}\left\|\left(x_{n}\right)\right\|_{p^{\prime}, \infty} \leq C\|f\|_{\operatorname{Bloch}(X)}
$$

for $f(z)=\sum_{n=1}^{\infty} x_{n} z^{n}$.

Proposition 3.3. Let $X^{*}$ be a complex Banach space of Rademacher cotype $p^{\prime}$ and $Y$ be any Banach space. Then

$$
\left(\operatorname{Bloch}(X), \ell_{1}(Y)\right) \subset \ell\left(p^{\prime}, 1, \mathcal{L}(X, Y)\right) .
$$

Proof. Let $\left(T_{n}\right)$ be a sequence of operators in $\left(B \operatorname{loch}(X), \ell_{1}(Y)\right)$. Using a simple duality argument we get

$$
\left\|\sum_{n=1}^{\infty} \varepsilon_{n} T_{n}^{*}\left(y_{n}^{*}\right) z^{n}\right\|_{J_{1}\left(X^{*}\right)} \leq C
$$

for all $\varepsilon_{n} \in\{-1,1\}$ and $\left\|y_{n}^{*}\right\|=1$.

Now writing $\varepsilon_{n}=r_{n}(t)$ for $t \in[0,1]$ and $f_{t}(z)=\sum_{n=1}^{\infty} r_{n}(t) T_{n}^{*}\left(y_{n}^{*}\right) z^{n}$, we have

$$
\begin{aligned}
\int_{0}^{1}\left\|f_{t}\right\|_{J_{1}\left(X^{*}\right)} d t & =\int_{0}^{1} \int_{0}^{1}\left|M_{1}\left(f_{t}^{\prime}, r\right)\right| d r d t \\
& =\int_{0}^{1} \int_{0}^{2 \pi} \int_{0}^{1}\left|\sum_{n=1}^{\infty} n r_{n}(t) T_{n}^{*}\left(y_{n}^{*}\right) r^{n-1} e^{i(n-1) \theta}\right| d t \frac{d \theta}{2 \pi} d r \\
& \geq C \int_{0}^{1}\left(\sum_{n} n^{p^{\prime}}\left\|T_{n}^{*}\left(y_{n}^{*}\right)\right\|^{p^{\prime}} r^{n p^{\prime}}\right)^{1 / p^{\prime}} d r .
\end{aligned}
$$

Applying Lemma 2.2 for $\beta=p^{\prime}$ and $q=1 / p^{\prime}$, we obtain $\left(T_{n}^{*}\left(y_{n}^{*}\right)\right) \in$ $\ell\left(p^{\prime}, 1, X^{*}\right)$ uniformly for $\left\|y_{n}^{*}\right\|=1$. Hence $\left(T_{n}\right) \in \ell\left(p^{\prime}, 1, \mathcal{L}(X, Y)\right)$.

Combining now Propositions 3.2 and 3.3 we get our final corollary: 
Corollary 3.4. Let $H$ be a Hilbert space and let $Y$ be a Banach space. Then

$$
\left(\operatorname{Bloch}(H), \ell_{1}(Y)\right)=\ell(2,1, \mathcal{L}(X, Y))
$$

\section{References}

[1] J. M. Anderson, J. Clunie and C. Pommerenke, On Bloch functions and normal functions, J. Reine Angew. Math. 270 (1974), 12-37.

[2] J. M. Anderson and A. L. Shields, Coefficient multipliers of Bloch functions, Trans. Amer. Math. Soc. 224 (1976), 256-265.

[3] J. L. Arregui and O. Blasco, Convolution of three functions by means of bilinear maps and applications, Illinois J. Math. 43 (1999), 264-280.

[4] -, -, Multipliers on vector-valued Bergman spaces, Canad. J. Math. 54 (2002), $1165-1186$.

[5] —, - Bergman and Bloch spaces of vector-valued functions, Math. Nachr. 261/262 (2003), 3-22.

[6] G. Bennett, D. A. Stegenga and R. M. Timoney, Coefficients of Bloch and Lipschitz functions, Illinois J. Math. 25 (1981), 520-531.

[7] O. Blasco, Spaces of vector-valued analytic functions and applications, in: London Math. Soc. Lecture Note Ser. 158, Cambridge Univ. Press, Cambridge, 1990, 33-48.

[8] -, Operators on weighted Bergman spaces and applications, Duke Math. J. 66 (1992), 443-467.

[9] - A characterization of Hilbert spaces in terms of multipliers between spaces of vector valued analytic functions, Michigan Math. J. 42 (1995), 537-543.

[10] - Vector-valued analytic functions of bounded mean oscillation and geometry of Banach spaces, Illinois J. Math. 41 (1997), 532-558.

[11] -, Multipliers on spaces of analytic functions, Canad. J. Math. 47 (1995), 44-64.

[12] - Convolution by means of bilinear maps, in: Contemp. Math. 232, Amer. Math. Soc., Providence, RI, 1999, 85-103.

[13] -, Remarks on vector-valued BMOA and vector-valued multipliers, Positivity 4 (2000), 339-356.

[14] -, Vector-valued Hardy inequality and B-convexity, Ark. Mat. 38 (2000), 21-36.

[15] O. Blasco and A. Pełczyński, Theorems of Hardy and Paley for vector-valued analytic functions and related classes of Banach spaces, Trans. Amer. Math. Soc. 323 (1991), 335-367.

[16] J. Diestel, H. Jarchow and A. Tonge, Absolutely Summing Operators, Cambridge Univ. Press, Cambridge, 1995.

[17] P. L. Duren, Theory of $H^{p}$ Spaces, Academic Press, New York, 1970.

[18] J. García-Cuerva, K. S. Kazarian, V. I. Kolyada and J. L. Torrea, Vector-valued Hausdorff-Young inequality and applications, Russian Math. Surveys 53 (1998), $435-513$.

[19] M. Jevtić and M. Pavlović, Coefficient multipliers on spaces of analytic functions, Acta Sci. Math. (Szeged) 64 (1998), 531-545.

[20] C. N. Kellogg, An extension of the Hausdorff-Young theorem, Michigan Math. J. 18 (1971), 121-127.

[21] M. Mateljević and M. Pavlović, $L^{p}$-behaviour of the integral means of analytic functions, Studia Math. 77 (1984), 219-237. 
[22] B. Maurey et G. Pisier, Séries de variables aléatoires vectorielles indépendantes et propriétés géométriques des espaces de Banach, ibid. 58 (1976), 45-90.

[23] J. Peetre, Sur la transformation de Fourier des fonctions à valeurs vectorielles, Rend. Sem. Mat. Univ. Padova 42 (1969), 15-46.

[24] M. Talagrand, Cotype of operators from $C(K)$, Invent. Math. 107 (1992), 1-40.

[25] - Cotype and (q,1)-summing norm in a Banach space, ibid. 110 (1992), 545-556.

[26] P. Wojtaszczyk, Banach Spaces for Analysts, Cambridge Univ. Press, Cambridge, 1991.

[27] K. Zhu, Operators on Bergman Spaces, Dekker, New York, 1990.

Departamento de Análisis Matemático

Universidad de Valencia

46100 Burjassot

Valencia, Spain

E-mail: oblasco@uv.es

Received March 25, 2003

Revised version August 28, 2003 